\title{
Feasibility studies of production and electromagnetic de- cay studies of hyperons for HADES
}

\author{
Krzysztof Nowakowski ${ }^{1, *}$ and Joanna Kubos ${ }^{2}$ \\ for the HADES Collaboration \\ ${ }^{1}$ Jagiellonian University, Kraków, Poland \\ ${ }^{2}$ Institute of Nuclear Physics PAS, Krakow, Poland
}

\begin{abstract}
The spectrum of excited states of single and double strange hyperons is only poorly known. Their internal structure is controversially discussed within quark, bag and molecular models. The most famous example is $\Lambda(1405)$. In this context radiative decays are predicted to be an ideal tool to discriminate between various predictions. Moreover, $\Xi^{-}$production yield measured by HADES in $\mathrm{Ar}+\mathrm{KCl} @ 1.76 \mathrm{GeV}$ and $p+\mathrm{Nb} @ 3.5 \mathrm{GeV}$ experiments strongly overshoots theory predictions and calls for more detailed studies in elementary collisions. In our poster we show results of the feasibility studies for the HADES detector of the cascade production close to the threshold and also the radiative decays of the excited hyperon states. Three bench mark channels: $p p \rightarrow \Lambda(1520) K^{+} p \rightarrow \Lambda e^{+} e^{-} K^{+} p, p p \rightarrow \Lambda(1405) K^{+} p \rightarrow \Lambda e^{+} e^{-} K^{+} p$ and $p p \rightarrow \Sigma(1385) K^{+} p \rightarrow \Lambda e^{+} e^{-} K^{+} p$, at beam energy $E_{k}=4.5 \mathrm{GeV}$ have been put into extensive simulation studies together with the most significant background channels. We will show the results including expected count rates and signal to background ratios.
\end{abstract}

\section{HADES detector}

HADES (Ref. [1]) is a versatile apparatus especially designed for di-lepton spectroscopy in proton-proton, $\pi$-nuclei, proton-nuclei and heavy ion induced reactions. It consists of following sub-detectors: a hadron blind RICH (Ring-Imaging CHerenkov detector) - for lepton identification; MDC (Multi-wire Drift Chamber) and magnetic field for tracking and momentum measurement and TOF (Time Of Flight) detector which provides information about particles velocity. In close future HADES will be significantly upgraded, what will increase its abilities to measure hyperons. The most important development from this point of view is a new Forward Detector which will cover polar angle, below $7^{\circ}$ where a significant fraction of protons from hyperon decays are emitted. Also a new, based on PMTs, read-out for RICH detector, will play a very important role for particular electromagnetic decays because it will significantly increase leptons detection efficiency. Another, already assembled detector, is an electromagnetic calorimeter featuring 978 lead glass modules. A scheme of HADES and its ongoing upgrades is presented in Fig. 1.

\footnotetext{
*e-mail: k.nowakowski@doctoral.uj.edu.pl
} 


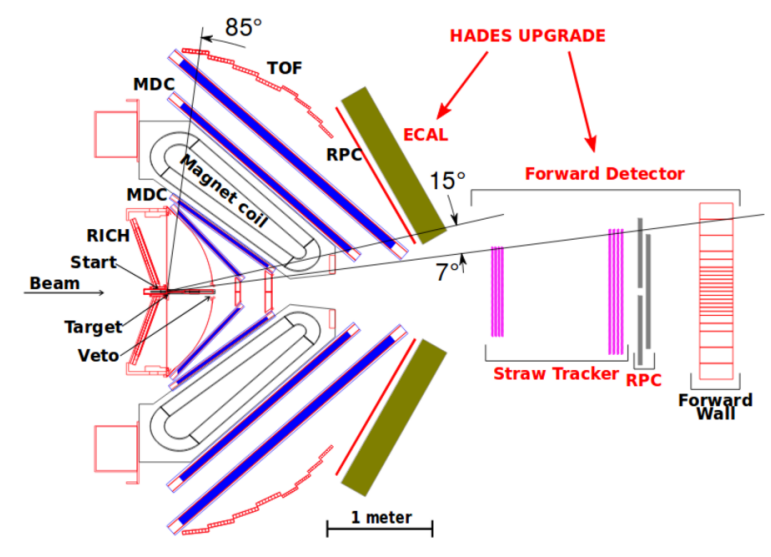

Figure 1. A sketch of the HADES detector (a side view in a vertical plane). The ongoing upgrades are labeled by read captions.

\section{Simulations}

Feasibility studies of the electromagnetic decays of hyperons have been performed by means of Monte Carlo simulations. Except the signal channels we also simulated and analyzed the most prominent background channels, to see how they influence reconstruction of the decays. A list of all simulated channels for $\Lambda(1520) \Lambda(1405)$ and $\Sigma(1385)$ decay, together with the background channels is shown in Table 1. Cross sections for each channel were estimated based on Landolt-Börnstein database [2] and publications: [3-5]. Branching ratio for Dalitz decays have been estimated from radiative decays $\left(\mathrm{BR}_{Y \rightarrow \Lambda e^{+} e^{-}}=\mathrm{BR}_{Y \rightarrow \Lambda \gamma} \times \frac{1}{137}\right)$. Simulations have been performed using two kinds of frameworks: PLUTO [6] as an event generator, and HGeant/HYDRA package to track particle propagation through the detector, and to track reconstruction, like for real data. The full detector geometry was implemented in HGEANT, including a new forward tracker.

Table 1. List of simulated reactions. Rows from 1 to 3: signal channels (including BR for Dalitz decay), 4-10: background channels. Background reactions are divided into three groups,

4-6: multiple pion production with at least one $\pi^{0}, 7-9: \Lambda(1115)$ associated with di-lepton source, 10: $\Lambda(1520)$ decay modes different then Dalitz decay.

\begin{tabular}{clcclc}
\hline No. & $p p \rightarrow$ & cross section $[\mu \mathrm{b}]$ & No. & $p p \rightarrow$ & cross section $[\mu \mathrm{b}]$ \\
\hline 1 & $p K^{+} \Lambda(1520)\left[\Lambda(1115) e^{+} e^{-}\right]$ & $8.3 \times 10^{-3}$ & 6 & $p n 2 \pi^{+} \pi^{-} \pi^{0}$ & 20 \\
2 & $p K^{+} \Lambda(1405)\left[\Lambda(1115) e^{+} e^{-}\right]$ & $8.8 \times 10^{-4}$ & 7 & $p K^{+} \Lambda(1115) \pi^{0}$ & 100 \\
3 & $p K^{+} \Sigma(1385)\left[\Lambda(1115) e^{+} e^{-}\right]$ & $7 \times 10^{-3}$ & 8 & $p K^{+} \Lambda(1115) 2 \pi^{0}$ & 20 \\
4 & $p p \pi^{+} \pi^{-} \pi^{0}$ & 1840 & 9 & $p K^{+} \Lambda(1115) 3 \pi^{0}$ & 7 \\
5 & $p p \pi^{+} \pi^{-} 2 \pi^{0}$ & 300 & 10 & Other decays of $\Lambda(1520)$ & 130
\end{tabular}

\section{Performed analysis}

Hyperon Dalitz decays have two main features utilized in the reconstruction. Their natural widths are relatively narrow, what makes identification much easier and their decays involve $\Lambda$ ground state, whose mean lifetime is $2.63 \times 10^{-10} \mathrm{~s}(c \tau=7.89 \mathrm{~cm})$. The latter means that 
it mostly decays out of the target. Therefore, a set of cuts enhancing out-off reaction vertex decays have been applied on proton-pion candidate pairs to suppress abundant multi-pion production. Putting minimal distance between $\pi^{-}$and proton tracks we can further reduce the background. Finally, a narrow cut on proton-pion invariant mass was applied to select $\Lambda(1115)$ candidates.

Summarizing, three cuts were used in the analysis: (1) distance between $\pi^{-}$and proton track required to be less than $20 \mathrm{~mm}$, (2) $\Lambda(1115)$ decay vertex should be at least $1 \mathrm{~cm}$ from the end of the target and (3) invariant mass of $\pi^{-}$proton pair $1105 \mathrm{MeV} / \mathrm{c}^{2}<M_{\pi^{-}} p<$ $1125 \mathrm{MeV} / \mathrm{c}^{2}$. Finally $\Lambda$ candidates were combined with $e^{+} e^{-}$tracks matched in RICH and MDC. Using such selection criteria we obtained results shown in Fig. 2. A combinatorial background has been estimated and subtracted from a reconstructed $\Lambda e^{+} e^{-}$sample using a like-sign di-lepton pairs. A good signal to combinatorial background ratio (1.7 for masses $M_{e^{+} e^{-}}>140 \mathrm{MeV}$ ) proves that it is possible to measure hyperon electromagnetic decays with HADES.

Assuming a luminosity $2 \times 10^{31} \mathrm{~cm}^{-2} \mathrm{~s}^{-1}$, equivalent to a beam rate of $10^{8}$ particles/s, a target with density of protons $2 \times 10^{23} \mathrm{~cm}^{-2}$, cross-section written in Tab. 1, and the accelerator duty cycle $50 \%$ we expect around 6800 hyperon Dalitz decays per day. In simulation a reconstruction efficiency of $0.5 \%$ was achieved, which gives an expected count rate of 34 hyperon decays per day.
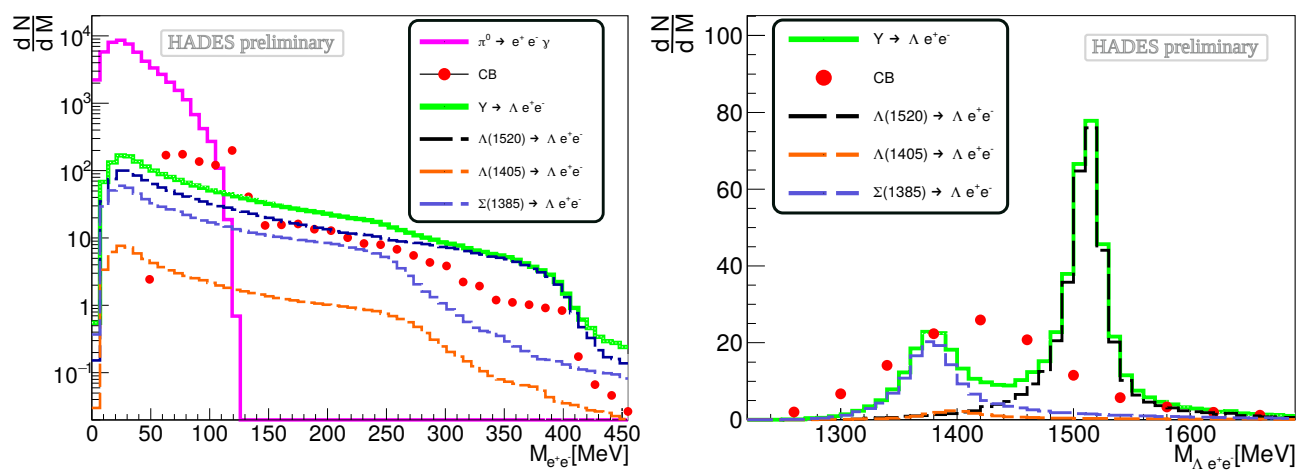

Figure 2. Left: di-lepton invariant mass spectrum. Thee green solid line shows the sum of all signal channels, dashed lines show each channel separately (see legend), red dots came from combinatorial background, magenta solid line is a contribution from $\pi^{0}$ Dalitz decay. Right: invariant mass for pairs $\Lambda^{0}$ - di-lepton, for $M_{e^{+} e^{-}}>M_{\pi^{0}}$. Color code the same like in left.

This work has been supported by following grants founded by National Science Centre:

2016/23/P/ST2/04066 POLONEZ

2017/25/N/ST2/00580 PRELUDIUM

\section{References}

[1] G. Agakichiev, et al. (HADES collaboration), Eur. Phys. J. A 41, 243 (2009)

[2] G. Höhler, ed., Landolt-Börnstein - Group I Elementary Particles, Nuclei and Atoms (Springer, 1983)

[3] J. Adamczewski-Musch, et al. (HADES Collaboration), Phys. Rev. C 95, 015207 (2017) 
[4] G. Agakishiev, et al. (HADES Collaboration), Phys. Rev. Lett. 114, 212301 (2015)

[5] J. Adamczewski-Musch, et al. (HADES Collaboration), Physics Letters B 781, 735 (2018)

[6] I. Frohlich, et al., PoS ACAT, 076 (2007) 